\title{
Two-loop renormalisation of non-Abelian gauge theories in $4 D$ Implicit Regularisation
}

\author{
Adriano Cherchiglia, ${ }^{a}$ Dafne Carolina Arias-Perdomo, ${ }^{a, *}$ Alexandre Vieira, ${ }^{b}$ Marcos \\ Sampaio $^{a}$ and Brigitte Hiller ${ }^{c}$ \\ ${ }^{a} \mathrm{CCNH}$, Universidade Federal do $\mathrm{ABC}$, \\ Santo André, SP 09210-580, Brazil \\ ${ }^{b}$ Universidade Federal do Triângulo Mineiro, \\ Iturama, MG 38280-000, Brazil \\ ${ }^{c}$ CFisUC, Department of Physics, University of Coimbra, \\ 3004-516 Coimbra, Portugal \\ E-mail: adriano.cherchiglia@ufabc.edu.br, carolina.perdomo@ufabc.edu.br, \\ alexandre.vieira@uftm.edu.br, marcos.sampaio@ufabc.edu.br, \\ brigitte@fis.uc.pt
}

The data collected at the LHC does not indicate significant deviations from the predictions of the Standard Model (SM). Taking into account that precision observables can be predicted already at two and three loops, it is evident the necessity to develop stringent tests of selfconsistency of the SM. In this work, we compute the 2-loop $\beta$-function of pure Yang-Mills and quantum chromodynamics using the background field method in a fully quadridimensional setup using Implicit Regularisation (IREG). In IREG the renormalisation constants are defined as basic divergent integrals. Finally, an algorithm for the automated calculation of the $\beta$-function was developed in Mathematica.

*** The European Physical Society Conference on High Energy Physics (EPS-HEP2021), ***

*** 26-30 July $2021 * * *$

*** Online conference, jointly organized by Universität Hamburg and the research center DESY ***

${ }^{*}$ Speaker 


\section{Motivations}

Among different proposals to gain in efficiency in higher order perturbative calculations, new regularisation schemes prove to be a viable alternative for theoretical predictions [1].

In this scenario, we pursued 2-loop corrections in non-abelian theories using Implicit Regularisation (IREG). Our main objective was to understand how to wisely remove the ultraviolet divergences (UV-div) when they arise at 2-loop order for non-abelian gauge theories with IREG. We organize our work as follows: in section 2 we present a very brief overview of the traditional regularisation schemes and IREG, while in section 3 we discuss the algorithm that we are implementing in our work for calculating the $\beta$-function at 2-loop for non-abelian theories. In section 4 we present our results. Finally, we conclude in section 5 .

\section{Regularisation schemes and the implicit regularisation formalism}

In this section we do a brief description of the general ideas of the traditional dimensional methods and the IREG method. For further details, we refer to [1-3].

Traditional dimensional schemes (DS) are the most used in QFT. They are based on analytical continuations of the space from 4 to $\mathrm{d}$ dimensions. We can distinguish two forms of dimensional schemes. The first is dimensional regularisation (CDR) which analytically continues the integral into $d=4-2 \epsilon$. CDR faces issues associated with SUSY breaking since altering the number of space-time dimensions leads to an imbalance between the fermionic and the bosonic degrees of freedom. With this in mind, some variants of CDR such as dimensional reduction (DRED) [4] have been developed. In DS, UV-div manifest as poles of form $1 / \epsilon^{n}$.

The issues of UV-div in $N^{2} L O$ and $N^{3} L O$ would be greatly simplified in a purely 4-dimensional scheme. IREG [5] works in momentum space and stays in 4 dimensions. With this procedure by applying a mathematical identity at the integrand level, the UV-div of any Feynman integral does not depend on physical parameters such as external momenta or masses. The objective of IREG is to write the UV-div in terms of the Basic Divergent Integrals (BDI). For example, the UV divergent part of a general 1-loop Feynman amplitude is proportional to

$$
I_{\log }\left(\Lambda^{2}\right) \equiv \int_{-\infty}^{+\infty} \frac{d^{4} k}{(2 \pi)^{4}} \frac{1}{\left(k^{2}-\Lambda^{2}\right)^{2}}
$$

where $\Lambda^{2}$ plays the role of the renormalisation group scale in the method.

\section{IREG to 2-loop order: non-abelian theories and the background field method}

In the direction of computing amplitudes in QFT, it is useful to develop routines for automated calculations. Thus, we developed a code in Mathematica to find the $\beta$-function coefficients of gauge couplings. This code works at a 2-loop level based on a bare algorithm developed in Ref. [3].

In the first stage of this algorithm, we use the Feynman diagram generator FeynArts. This package allows controlling the production of the necessary topologies of interest. Yet, it not only 
generates the topologies, but it can also create the Feynman amplitudes of the processes under study. In the second stage, we make the necessary simplifications and reductions for the integrals. These simplifications depend on the regularisation scheme used. In the final stage, we evaluate the integrals according to the regularisation method used. Finally, we use the definition of the $\beta$-function to compute it at this stage. For a summary and outline of all these stages see Fig. 1.

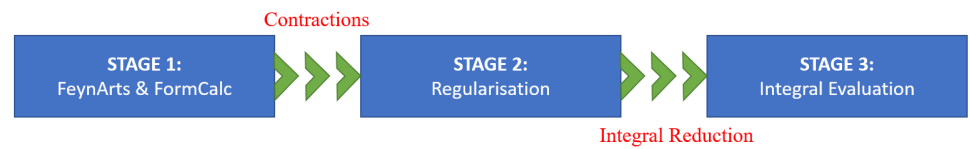

Figure 1: Algorithm used for the automatic calculation of the $\beta$-function at 2-loop.

For the calculation of the 2-loop $\beta$-function in non-abelian gauge theories, we use the background field method (BFM) [6]. The BFM simplifies the computation since only 2-point functions are needed. For example, in pure Yang-Mills, this implies that only topologies as the ones in Fig. 2 can appear.

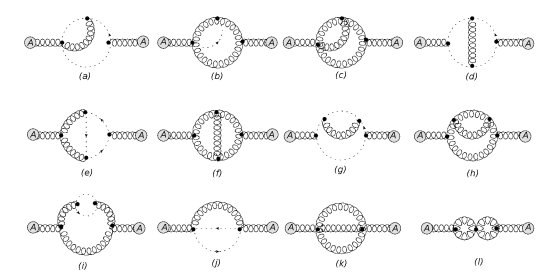

Figure 2: The 2-loop corrections to the 2-point function of the background field for pure Yang-Mills.

\section{Results: $\beta$-function for non-abelian theories to 2-loop order}

In this last section, we present the gauge coupling $\beta$-function up to 2-loop order obtained within the IREG framework for the non-abelian theories. As standard, one can define [3] the expansion of the $\beta$-function in the adimensional coupling constant as

$$
\beta=-g_{R}\left[\beta_{0}\left(\frac{\tilde{g}_{R}}{4 \pi}\right)^{2}+\beta_{1}\left(\frac{\tilde{g}_{R}}{4 \pi}\right)^{4}\right]
$$

where $g_{R}$ is the (renormalised) gauge coupling of the theory considered and $\tilde{g}_{R}$ is the (renormalised) adimensional coupling constant.

Finally, by using the $\beta$-function definition and the results collected from the diagrams, we can obtain the 1 and 2-loop contributions for the gauge $\beta$ coupling in pure Yang-Mills

$$
\begin{gathered}
\left.\beta\right|_{\mathrm{DS}}=-g_{R}\left[\frac{11}{3} C_{A}\left(\frac{\tilde{g}_{R}}{4 \pi}\right)^{2}+\frac{34}{3} C_{A}^{2}\left(\frac{\tilde{g}_{R}}{4 \pi}\right)^{4}\right] ; \\
\left.\beta\right|_{\text {IREG }}=-g_{R}\left[\frac{11}{3} C_{A}\left(\frac{\tilde{g}_{R}}{4 \pi}\right)^{2}+\frac{34}{3} C_{A}^{2}\left(\frac{\tilde{g}_{R}}{4 \pi}\right)^{4}\right] ;
\end{gathered}
$$


and in QCD

$$
\begin{gathered}
\left.\beta_{0}\right|_{\text {IREG }}=11-\frac{2}{3} n_{f} ;\left.\quad \beta_{0}\right|_{\mathrm{CDR}}=11-\frac{2}{3} n_{f} ; \\
\left.\beta_{1}\right|_{\text {IREG }}=102-\frac{38}{3} n_{f} ;\left.\quad \beta_{1}\right|_{\mathrm{CDR}}=102-\frac{38}{3} n_{f} .
\end{gathered}
$$

These results are in agreement with the well known first two coefficients of the $\beta$-function which are universal in mass-independent renormalisation schemes. Notice also that the $\beta$-function is finite as expected.

\section{Conclusion and perspectives}

In this work, we presented the $\beta$-function to 2-loop order of the above-mentioned non-abelian theories. This provides for a consistency test of the technique. To complete the 2-loop project with IREG, we are performing the calculation of the 2-loop quark mass anomalous dimension

$$
\gamma_{m}^{I R \bar{E} G}\left(\Lambda^{2}\right)=2 \frac{\Lambda^{2}}{m^{I R \bar{E} G}} \frac{\partial m^{I R \bar{E} G}}{\partial \Lambda^{2}}=-\beta^{I R \bar{E} G}\left(\Lambda^{2}\right) \frac{\partial \ln Z_{m}^{I R \bar{E} G}}{\partial g^{I R \bar{E} G}}
$$

in QCD within IREG in a mass-independent regularisation scheme $(I R \bar{E} G)$.

\section{Acknowledgments}

M.S. acknowledges a research grant from CNPq (Conselho Nacional de Desenvolvimento Científico e Tecnológico - 303482/2017-6). We acknowledge support from Fundação para a Ciência e Tecnologia (FCT) through the projects UID/FIS/04564/2020 and CERN/FIS-COM/0035/2019, and the networking support by the COST Action CA16201. This study was financed in part by the Coordenação de Aperfeiçoamento de Pessoal de Nível Superior - Brasil (CAPES) - Finance Code 001.

\section{References}

[1] C. Gnendiger, et al., Eur. Phys. J. C 77 (2017) 471.

[2] W. J. Torres Bobadilla, G. F. R. Sborlini, P. Banerjee, S. Catani, A. L. Cherchiglia, L. Cieri, P. K. Dhani, F. Driencourt-Mangin, T. Engel and G. Ferrera, et al. Eur. Phys. J. C 81, no.3, $250(2021)$

[3] A. Cherchiglia, D. C. Arias-Perdomo, A. R. Vieira, M. Sampaio and B. Hiller, Eur. Phys. J. C 81 (2021) no.5, 468

[4] W. Siegel, 84(1979) 193; idem, 94(1980) 37.

[5] D. C. Arias-Perdomo, A. Cherchiglia, B. Hiller and M. Sampaio, Symmetry 13 (2021) no.6, 956

[6] L. Abbott, Acta Phys. Polon. B 13, 33 (1982) CERN-TH-3113. 